\title{
Application and Design of Patient Temperature Acquisition System Based on Wireless Sensor Network
}

\author{
https://doi.org/10.3991/ijoe.v13i05.7046 \\ Huijie Ding \\ The Open University of Guangdong, Guangdong, China \\ sbce893809@126.com
}

\begin{abstract}
The purpose of this study is to achieve large-scale real-time acquisition and monitoring of the patient's body temperature, for noticing abnormal phenomenon of temperature in time. In the ward, arrange ZigBee wireless temperature monitoring network, realize the ward patients temperature measurement and gathering of temperature related clinical data, achieve the temperature monitoring network through the serial RS232 and PC communication, and $\mathrm{PC}$ receives, displays, and processes the data through the serial port. The results showed that the system can also realize the acquisition, transmission and monitoring of a plurality of temperature signals, and send out the alarm in abnormal temperature. As a result, the system can be widely used in clinical measurement, suitable for popularization in large hospital with a large number of patients.
\end{abstract}

Keywords-ZigBee; temperature monitoring network; body temperature

\section{Introduction}

Body temperature plays an important role in patient care and clinical diagnosis. At present, temperature monitoring of patients is mainly the artificial timing measurement method. Medical staffs measure the temperature every day in certain time one by one through the traditional mercury thermometer. This cannot achieve large-scale measurement, and cannot be real-time monitoring of temperature changes of patients, and cannot find the abnormal in time, so it may lead to that the patients miss the best timing of treatment, while frequent contact, for infectious diseases, is easy to cause the medical staff infection, and spends a lot of manpower [1]. In many cities and hospitals in the United States, it has begun to ban the use of mercury thermometer, and some large chain stores have also changed the electronic thermometer. Electronic thermometers on the market are mostly thermistor and infrared type, but electronic thermometer that can monitor the body temperature for a long time is rare [2]. In consequence, it is necessary to realize the large-scale real-time monitoring of patients, without the need for frequent contact measurement. In recent years, wireless technology has been gradually applied to the field of medical care. Wireless sensor network is used in the field of human physiological parameters monitoring with its own characteristics. 
Paper-Application and Design of Patient Temperature Acquisition System Based on Wireless Sensor...

This research, based on the ZigBee wireless sensor network technology with low power consumption, takes the system CC2530 chip on the chip as the core, and DS18B20 as the temperature sensor, and designs and implements real-time monitoring system of clinical patient's temperature based on ZigBee. The system can meet the large-scale real-time monitoring for patients without frequent contact; in addition, the system also has low power consumption, miniaturization, strong expansibility and so on characteristics.

\section{State of the art}

At present, the hospital ward monitoring system mostly transfers to the monitoring center through the cable, so it needs for wiring, walling or ceiling wiring cumbersome work, and the mobility is not strong. In particular, the patient's body temperature needs manual measurement.

In recent years, due to people's pursuit of intelligence, wireless sensor networks are developing rapidly. Wireless sensor network is a new technology that combines the technologies in many fields. Wireless sensor network consists of many wireless sensor nodes, and each sensor node in the case of electricity, can obtain the relevant data in the wireless network. The data acquisition module is different that it can realize the acquisition of different data parameters. With the increasing integration of hardware and mature of technology, sensor node volume decreased gradually and the cost is reduced, so the construction cost of wireless sensor network is decreased. Compared with the traditional networks, wireless sensor networks have large-scale, selforganizing, dynamic reconfigurable information system, data centric and so on features, so wireless sensor network technology has been extended to all areas, including medical and health care field [3].

The application value of wireless sensor network is embodied in: (1) mobility. Because there are no cable restrictions, users can work in different places, and network users can access to real-time information resources anywhere. (2) no need for wiring. The installation is easy, and it can greatly shorten the construction time. (3) flexible networking. Wireless sensor networks can consist of a variety of topologies, and it is easy to expand from a small number of users to peer to peer network infrastructure [4].

At present, there are 5 popular wireless network technology standards: ZigBee, Wi-Fi, Bluetooth, Ultra Wide Band, and NFC [5].

ZigBee is a new wireless network technology with short distance, low rate, low power consumption and high communication efficiency. It is based on the IEEE 802.15 .4 , mutually coordinating in the thousands of tiny sensors to realize the communication between each other. These sensors require only a small amount of energy to relay the data from one sensor to another via radio waves, creating conditions for the ubiquitous network.

In summary, the traditional patient body temperature collection method has a great measurement error. The feedback of the system is not timely. The workload of medical staff is large. In view of the above shortcomings, this paper designed a set of wire- 
less sensor network technology based on the patient temperature acquisition system. In the system, we use a small size and low power consumption of the chip, so as to achieve the sensor node miniaturization and long working hours. Business service platform software uses a four-tier architecture, and reserve the interface of different sensor nodes and different applications. The system can not only be applied to the hospital, but also can be applied to the patient's health care. The system has reserved the interface which is connected with the hospital system. It can be easily embedded in the system platform of the hospital.

\section{Methods}

\subsection{System overall structure design}

Body temperature measurement is one of the basic nursing works in hospital, which needs to consume a lot of manpower and material resources. Wireless temperature monitoring system of this paper is to use the terminal acquisition node worn by the tester, to measure body temperature data. Wireless temperature monitoring system consists of a coordinator data aggregation uploading and command sending node, routing data and relay node, and terminal acquisition node.

The system takes the ward for the monitoring unit. Set wireless temperature monitoring network based on ZigBee technology in each ward building, and clinical patients use mobile temperature terminal node for the measurement of body temperature. Body temperature data makes instructions and data transmission and gathering through the ZigBee network, and finally accesses to the computer of the nursing station on each floor through the serial of the coordinator. Then, temperature data will be displayed in the computer accessed to, which is convenient for nursing staff observation, analysis, and processing temperature data. At the same time, set the threshold temperature, if the temperature of terminal nodes wearer's body temperature is higher or lower than the temperature threshold value, it will send an alarm signal, for timely detection of disease. According to the characteristics of the temperature monitoring system, the design of this paper adheres to the principle of miniaturization and modularization, the temperature information collection node must be able to work independently, and keep the system stable when there are new nodes joining or exiting the network. At the same time, it is necessary to reduce the power consumption for the long-term working of the terminal node, which is mainly realized by making the terminal node regularly in the working and sleeping state.

In view of the above demand analysis, this system takes the clinical patient's body temperature as the measuring object, and it can satisfy the large-scale measurement. The system is composed of four parts: the terminal node, the router node, the coordinator node and the PC. The terminal node, the router node and the coordinator node consist of the wireless sensor network of the system. Each patient is assigned a terminal acquisition node, and a routing node is arranged in the appropriate position of the ward, the corridor and so on, and each office building is provided with a coordinator and a PC to form a separate network. The block diagram is shown in figure 1. 


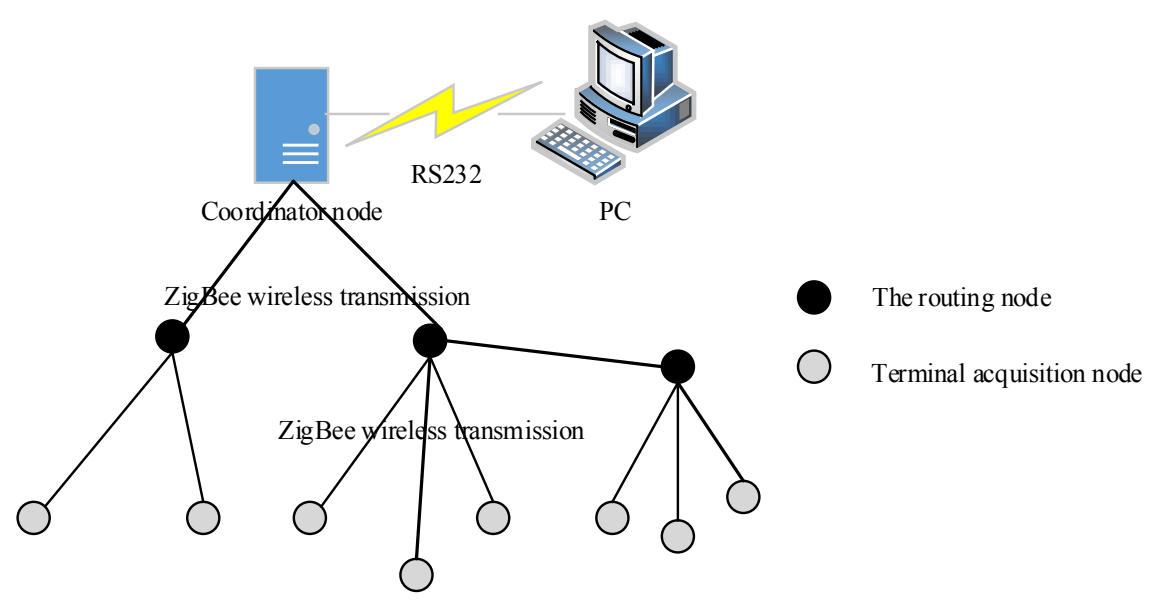

Fig. 1. System structure diagram

ZigBee based temperature monitoring system network nodes are divided into three kinds: the terminal acquisition node, the router node and the coordinator. Because the data in the system for the transmission process is the temperature data from the coordinator to the terminal acquisition node sending commands data and that from the terminal acquisition node to the coordinator, it will be helped by the intermediate relay of the routing nodes, and there is no need for mutual communication among nodes. In this way, we consider the selection of tree network topology as the network structure of the system.

\subsection{System hardware design}

There are 3 kinds of network nodes in the temperature monitoring system based on ZigBee: the terminal acquisition node, the routing node and the coordinator node. A network is composed of 1 coordination device, a plurality of routing nodes and a plurality of terminal acquisition nodes. These 3 kinds of nodes are the basic equipment to compose of the wireless sensor network. There is no difference in the hardware of the 3 types of nodes, and its functional properties are determined by the software compilation. 3 types of nodes of the system use CC2530 chip of TI Company as the core chip. CC2530 chip is a real SoC, $2.4 \mathrm{G}$ frequency. It can realize wireless communication and data processing with the circuit composed of a CC2530 chip and a few peripheral electronic components.

The main functions of the terminal acquisition node include state indication, data acquisition, and cooperation with the routing node to achieve the transmission of body temperature data, and it reduces the energy consumption as much as possible in the case of ensuring the working performance. The block diagram of the terminal acquisition node is shown in figure 2. The single bus digital temperature sensor DS18B20 used in the temperature monitoring module system is produced by DALLAS company. Compared with the thermal resistance, the tested temperature can be read directly, the programmable resolution is $9 \sim 12$ bit, the measurement results directly output 
digital temperature signal, and transfer to CPU with the "bus line" serial, at the same time transmit CRC check code, having strong ability of anti-interference correction. The utility model has the advantages of small volume, and the utility model can meet the requirements of the portable temperature measurement in the clinic [6]. The voltage is applicable to a wide range $(3.0 \mathrm{~V} \sim 5.5 \mathrm{~V})$, the external power supply can be used, and the parasitic power supply can also be used, so the DS18B20 can make the system simpler. In order to ensure the accuracy of temperature measurement, temperature measurement can be carried out under the armpit, and the sensor can be fixed in the part of patients with rich blood flow. The design circuit of this part is shown in figure 3.

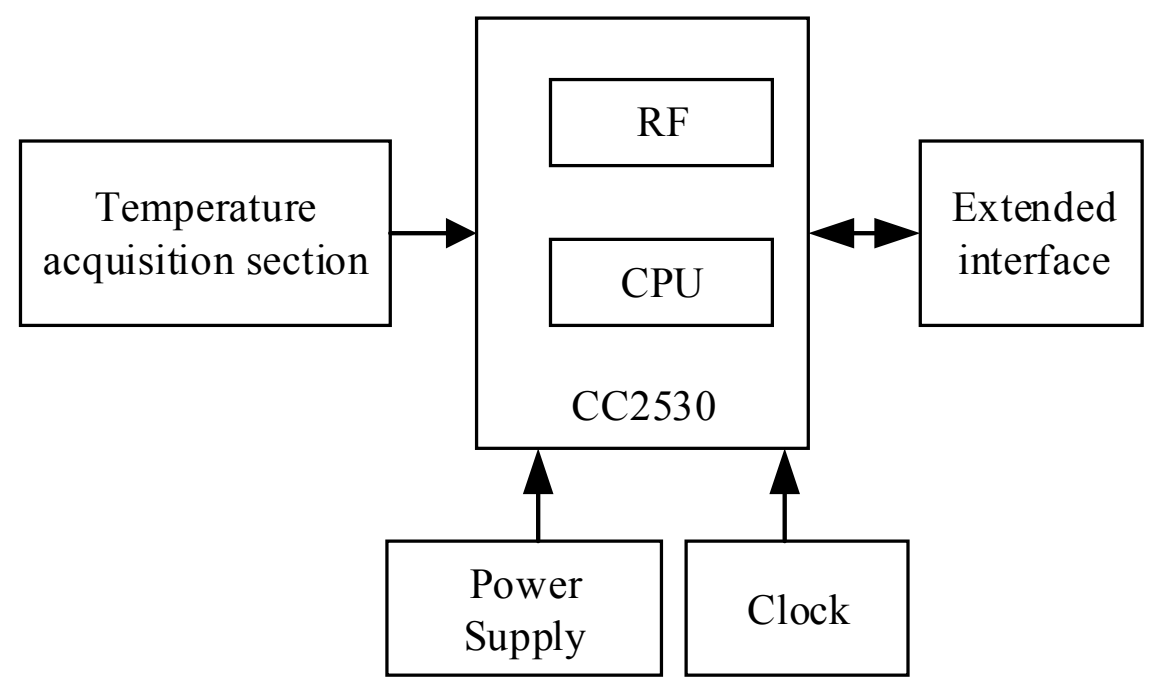

Fig. 2. Terminal acquisition node diagram

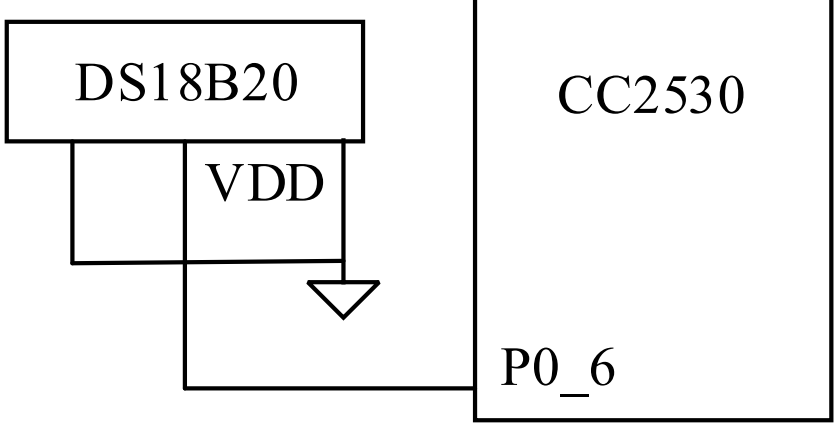

Fig. 3. Temperature acquisition circuit diagram 
The data transmission network is composed of the routing node and the coordinator node, which is mainly responsible for receiving the data packets sent by the acquisition node and make routing of the data packets, and transmit to the coordinator [7]. Routing node does not need to communicate with the PC machine, and the hardware part does not contain the serial part. The routing node block diagram is shown in figure 4.

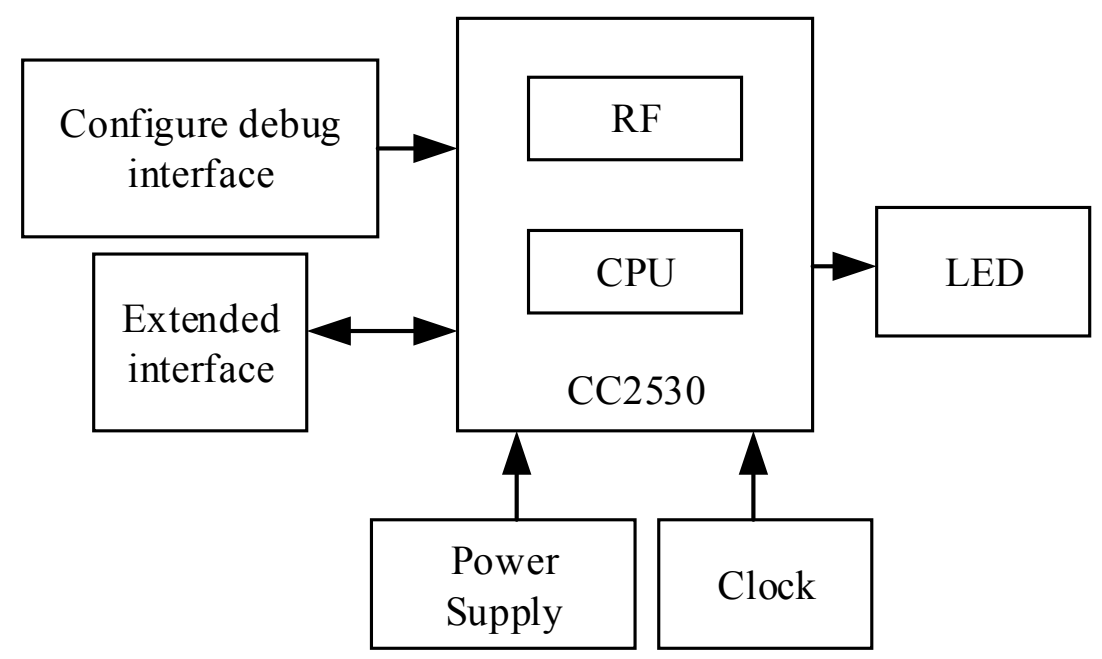

Fig. 4. Routing node diagram

The main function of the coordinator node is to launch the ZigBee wireless network, and receive the data sent by terminal acquisition node and routing node. It realizes the data aggregation and PC serial communication in the system, comparatively simple in the hardware structure. The coordinator node diagram is shown in figure 5.

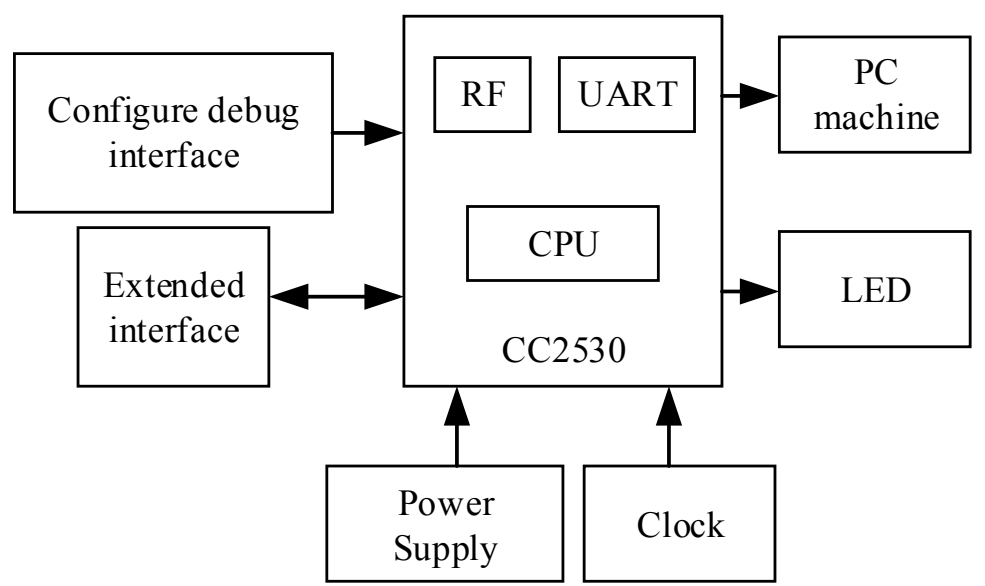

Fig. 5. Coordinator node diagram 
Paper-Application and Design of Patient Temperature Acquisition System Based on Wireless Sensor...

\subsection{System software design}

The software development environment of this system is IAR Embedded Workbench. IAR EW developed by IAR System is a powerful integrated development environment software, well received by the industry. As a software development platform, it can meet the needs of different users, and in allusion to different chip architectures, it has also developed corresponding different versions. This paper introduces the software design of coordinator node and terminal node in details.

In this system, the coordinator is responsible for the establishment of the network, data aggregation and serial communication with the host computer. The design uses non-beacon mode network, and the network channel uses the default configuration values in the f8wConfig.cfg file. After the coordinator is powered on, it does not require a network scan channel. When the coordinator constructs a network, it selects a random PANID, and after obtaining PANID, the new network starts running. After the coordinator constructs a network, it sends beacon frames to the nearby ZigBee device node by way of broadcasting in the network, and then enters the state of wireless monitoring [8]. After powering on of routing node and terminal acquisition node, it will firstly send a beacon request for searching network, and then receive the response from a coordinator, to send network request. After receiving allowance answer of a coordinator, it can join the network. At the same time, the coordinator takes it as the child node, to join its neighbor table, and assign a 16-bit full network unique short network address. The coordinator, after constructing the network, has been in the listening state, whether the nodes join and leave, whether it receives instruction information sent from the serial port, whether it receives the temperature data information uploaded by the terminal acquisition node or routing relay node, and it makes the appropriate treatment according to the information types.

The acquisition node software is used to provide the initialization of the system acquisition node, body temperature acquisition and communication with the parent node. After powering on, first of all, carry out initialization operation, then join the network, start collecting temperature information, and when the temperature is abnormal, timely upload data to the parent node. The parent node sends it to the coordinator node, and then transmits to the PC machine, which can ensure the timely detection and treatment of the abnormal body temperature; when the body temperature is normal and the parent node data requests an instruction, the temperature data is sent to the parent node after packaging. The node acquisition flow chart is shown in figure 6 . 


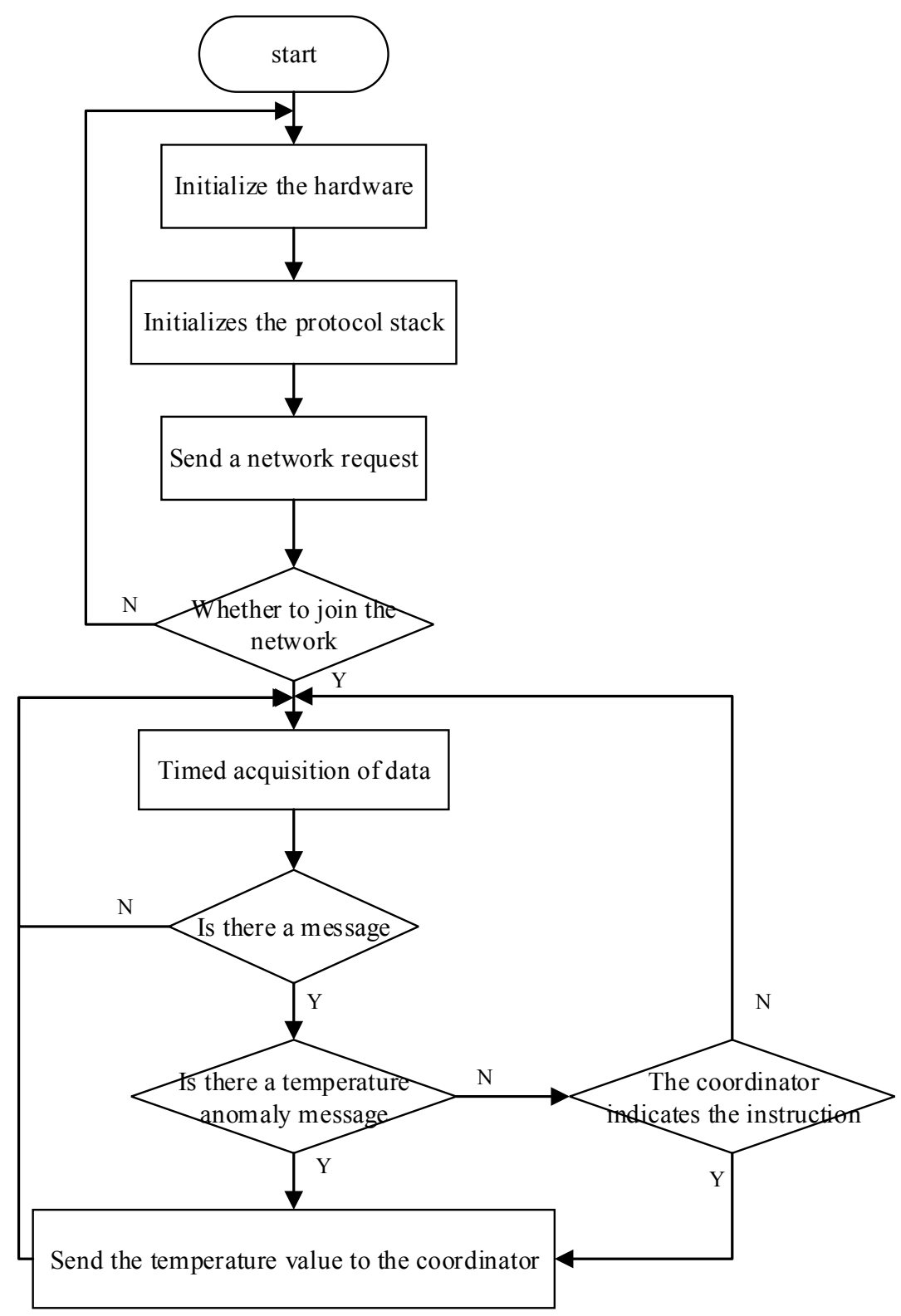

Fig. 6. Working flow chart of terminal acquisition node

\section{Experiment}

This experiment mainly includes two parts: ZigBee wireless network communication function test and system function test. 
Paper-Application and Design of Patient Temperature Acquisition System Based on Wireless Sensor...

\subsection{Experiment process}

In the ZigBee wireless sensor network communication function test, the tools needed to be used are the hardware device CC2531 USB Dongle, or CC Debuger (simulation download) connected to the CC2530-EB development board. The software tool is Texas Instruments Packet Sniffer, IEEE802.15.4/ZigBee protocol type, then it can capture ZigBee wireless data packet in the environment, and understand the working conditions of ZigBee network through the analysis of the data packet. In the upper computer, input acquisition commands and observe the host computer interface.

In the test of the system function, simultaneous test of the timing acquisition and traditional thermometer is used. When the temperature is normal, there is no feedback in the system; when the temperature exceeds the preset limit, the red light on the terminal acquisition module is flashing, and uploading the data of body temperature. When testing the timing acquisition, the time interval can be adjusted smaller, and similarly, use the method of simultaneous test of the traditional thermometer.

Instruction mode specific test methods are as follows:

The 3 terminal acquisition nodes are placed on the wrist of the 3 subjects, and then 3 subjects are arranged in two rooms. The coordinator is placed in the room with the resettlement of 2 subjects, and the routing node is placed in the room with the resettlement of q subject, so as to ensure the packet transmission. The normal body temperature range is set at 36 degrees $\mathrm{C} \sim 37 \mathrm{C}$, to test body temperature every ten minutes.

\subsection{Experimental results and discussion}

The test results of the communication function of the ZigBee wireless sensor network show that the receiving interface on the computer can display the temperature data uploaded by the terminal node, which indicates that the system operates normally, and has a good communication function.

The functional test of the system shows to transmit to the host computer through the serial port, and with the help of the serial debugging assistant software, when sending "begin" command from the serial port, it begins to display the body temperature data. It acquires the data once it sends the instruction every time, and the data is the average of the temperature for four times, then send "stop" instruction, and the data acquisition and upload is stopped. First of all, the test receiver uses the system for temperature measurement, and measures the temperature in the same position according to the conventional method. After repeated experiments comparison, the measurement error is in the range of \pm 0.1 DEG C. Some experimental data are shown in table 1 , in which the temperature measurement units are degrees Celsius. 
Table 1. System function test results

\begin{tabular}{|c|c|c|c|c|c|c|c|c|c|}
\hline \multirow{2}{*}{$\begin{array}{c}\text { Acquisi- } \\
\text { tion time }\end{array}$} & \multicolumn{3}{|c|}{ Subject 1 } & \multicolumn{3}{c|}{ Subject 2 } & \multicolumn{3}{c|}{ Subject 3 } \\
\cline { 2 - 11 } & $\begin{array}{c}\text { Terminal } \\
\text { collec- } \\
\text { tion }\end{array}$ & $\begin{array}{c}\text { Conven- } \\
\text { tional } \\
\text { collec- } \\
\text { tion }\end{array}$ & $\begin{array}{c}\text { System } \\
\text { feedback }\end{array}$ & $\begin{array}{c}\text { Terminal } \\
\text { collec- } \\
\text { tion }\end{array}$ & $\begin{array}{c}\text { Conven- } \\
\text { tional } \\
\text { collec- } \\
\text { tion }\end{array}$ & $\begin{array}{c}\text { System } \\
\text { feedback }\end{array}$ & $\begin{array}{c}\text { Terminal } \\
\text { collec- } \\
\text { tion }\end{array}$ & $\begin{array}{c}\text { Conven- } \\
\text { tional } \\
\text { collec- } \\
\text { tion }\end{array}$ & $\begin{array}{c}\text { System } \\
\text { feed- } \\
\text { back }\end{array}$ \\
\hline $9: 00$ & 36.43 & 36.40 & No & 36.98 & 37.02 & No & 36.78 & 36.81 & No \\
\hline $9: 10$ & 36.51 & 36.45 & No & 36.96 & 37.01 & No & 36.76 & 36.80 & No \\
\hline $9: 20$ & 36.53 & 36.55 & No & 37.02 & 37.01 & Alarmed & 36.79 & 36.84 & No \\
\hline $9: 30$ & 36.42 & 36.45 & No & 37.15 & 37.18 & Alarmed & 36.87 & 36.91 & No \\
\hline $9: 40$ & 36.31 & 36.40 & No & 36.97 & 36.99 & No & 36.79 & 36.75 & No \\
\hline $9: 50$ & 36.52 & 36.60 & No & 37.03 & 37.01 & Alarmed & 36.80 & 36.83 & No \\
\hline $10: 00$ & 36.51 & 36.55 & No & 36.96 & 36.98 & No & 37.01 & 36.94 & Alarme \\
& & & & & & & & & \\
\hline $10: 10$ & 36.50 & 36.50 & No & 36.95 & 36.98 & No & 36.96 & 36.89 & No \\
\hline $10: 20$ & 36.62 & 36.65 & No & 37.08 & 37.07 & Alarmed & 36.68 & 36.74 & No \\
\hline $10: 30$ & 36.54 & 36.60 & No & 37.18 & 37.18 & Alarmed & 36.89 & 36.80 & No \\
\hline
\end{tabular}

As can be seen from table 1 , the system sends out an alarm when the collected temperature exceeds the preset range.

The designed patient's body temperature acquisition system based on ZigBee wireless network is small, and has low energy consumption, easy for patients to wear, and the accuracy of temperature acquisition is high, suitable for being promoted in largescale hospitals with a large number of patients.

\section{Conclusion}

In this paper, the temperature monitoring system based on ZigBee technology is introduced from the aspects of system hardware and software implementation, and the terminal acquisition node is optimized in terms of miniaturization and low power consumption. The experimental results show that the system can meet the needs of measurement accuracy, and make real-time monitoring of patients in a large scale, which can be widely applied in clinical physiological signal measurement.

\section{References}

[1] Chang, K., Lee, J., Jun, C. H., \& Chung, H. (2016). Acquisition of a series of temperaturevaried sample spectra to induce characteristic structural changes of components and selection of target-descriptive variables among them for multivariate analysis to improve accuracy. Applied Spectroscopy Reviews, 51(7-9), 718-734. https://doi.org/10.1080/0570 4928.2016.1167069

[2] Gradel, K. O., Nielsen, S. L., Knudsen, J. D., Østergaard, C., Arpi, M., Jensen, T. G., ... \& Schønheyder, H. C. (2016). Seasonal Variation of Escherichia coli, Staphylococcus aureus, and Streptococcus pneumoniae Bacteremia According to Acquisition and Patient Charac- 
Paper-Application and Design of Patient Temperature Acquisition System Based on Wireless Sensor...

teristics: A Population-Based Study. infection control \& hospital epidemiology, 37(08), 946-953. https://doi.org/10.1017/ice.2016.89

[3] Kalpakis, K., Dasgupta, K., \& Namjoshi, P. (2014). Efficient algorithms for maximum lifetime data gathering and aggregation in wireless sensor networks. Computer Networks, 42(6), 697-716. https://doi.org/10.1016/S1389-1286(03)00212-3

[4] Shirazi, S. S. (2015). Energy Efficient Hierarchical Cluster-Based Routing for Wireless Sensor Networks. International Conference of Advanced Computer Science \& Information Technology (pp.61-69). https://doi.org/10.5121/csit.2015.51207

[5] Song, J. L., Cheng, W. L., Xu, Z. M., Yuan, S., \& Liu, M. H. (2016). Study on PID temperature control performance of a novel PTC material with room temperature Curie point. International Journal of Heat and Mass Transfer, 95, 1038-1046. https://doi.org/10.1016/ j.ijheatmasstransfer.2015.12.057

[6] Thomson, M. C., Mason, S. J., Phindela, T., \& Connor, S. J. (2016). Use of rainfall and sea surface temperature monitoring for malaria early warning system in botswana. American Journal of Tropical Medicine \& Hygiene, 73(1), 214-221.

[7] Wang, B., Wang, X., Chen, X., Hou, L., Xie, W., \& Pan, M. (2016). Acquisition of optimal operating temperature for epitaxial Si: P blocked-impurity-band detector based on temperature-dependent characteristics investigation. Optical and Quantum Electronics, 48(2), $1-8$

[8] Watson, J., Kavanagh, S., \& Nugent, S. (2016). High Precision Analog Multiplexers Enable Multi-Channel Data Acquisition in High Temperature Environments. Additional Papers and Presentations, 2016(HiTEC), 000035-000039.

\section{$7 \quad$ Author}

Huijie Ding is with the College of Information \& Engineering of The Open University of Guangdong, Guangdong, China (sbce893809@126.com).

Article submitted 05 April 2017. Published as resubmitted by the author 23 May 2017. 